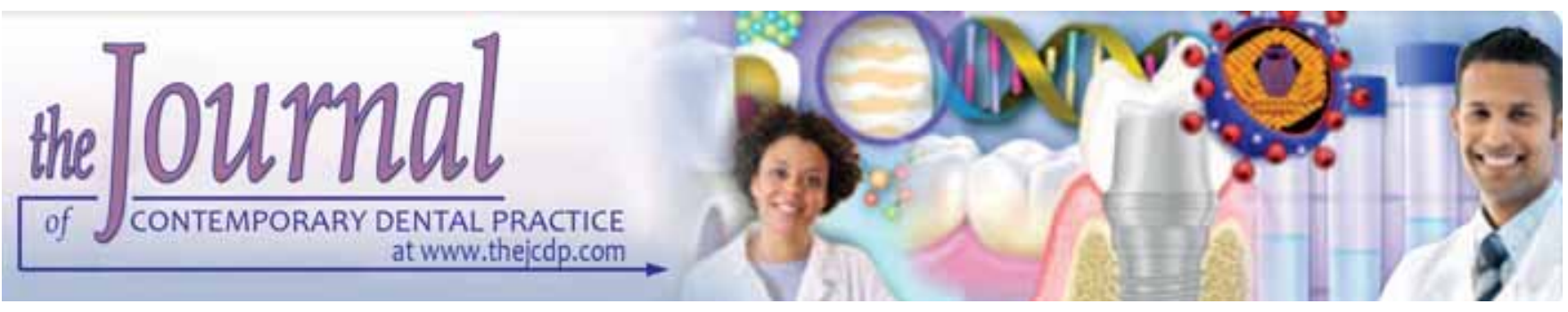

\title{
Microbiological Appraisal of Three Different Brands of Commercially Available Irreversible Hydrocolloid Impression Materials: An in vitro Study
}

\author{
Rakshith Guru, Mohammed Saleem, Rajeev Singh, Anand Patil
}

\begin{abstract}
Aim: To evaluate factory-sealed containers of three different commercially available irreversible hydrocolloid impression materials for their microbial contamination and the type of microorganisms present.
\end{abstract}

Materials and methods: Thirty-six measured samples were taken of each brand and placed on chocolate agar plates or in thioglycolate broth tubes and were incubated along with appropriate parallel controls. After incubation, colonies were enumerated and identified using standard microbiological methods.

Results: The three brands contained viable microorganisms in $90 \%$ of the samples. Samples from the top, middle and bottom layers had approximately equal contamination frequencies. The concentration of organism varied from 25 to 74 CFUs per gram of contaminated sample.

Conclusion and clinical significance: The samples contained viable microorganism which may be potentially dangerous to immunocompromised patients.

Keywords: Microbiological, Appraisal, Sealed, Irreversible hydrocolloid, Immunocompromised, Impression material.

How to cite this article: Guru R, Saleem M, Singh R, Patil A. Microbiological Appraisal of Three Different Brands of Commercially Available Irreversible Hydrocolloid Impression Materials: An in vitro Study. J Contemp Dent Pract 2011;12(1):35-40.

\section{Source of support: Nil}

Conflict of interest: None declared

\section{INTRODUCTION}

Nowadays, there has been an increasing emphasis on infection control in dentistry because of proliferation of infectious diseases and increase in number of medically compromised patients. Patients with HIV, cancer, transplants, autoimmune diseases are often immunocompromised and any microorganism they are exposed to must be considered as a potential pathogen. As it is impossible to determine the patients' immunological condition during each dental treatment, there is a need for standardized precautions and standards. The concept of universal precaution is advocated because neither all patients with infectious diseases are aware of their status nor can they be identified by medical history or physical examination. Therefore, it is important to prevent crosscontamination in every dental procedure.

The risk of transmitting pathogenic microorganisms via impressions has been considered as a topic of interest for a number of years. The possibility of contaminating immunocompromised patients in minor dental procedures, since they are susceptible to infections by microorganisms of low virulence, has been pointed out. ${ }^{1}$ Impression procedures frequently cause bleeding from the oral tissues. Considering that blood is a rich culture and microbial transportation medium, and that any rupture of skin integrity offers an opening for the entrance of potentially pathogenic microorganisms, there is a risk of accidental transmission of this infectious substrate to undesired places. ${ }^{2}$ Materials like irreversible hydrocolloid are often positioned against mucous membrane when used and could potentially result in inoculation of the patient. The use of contaminated impression materials may represent a potential hazard to immunocompromised patients.

The need for blocking this potentially infectious route between impression materials and patients is generally theoretical as information on the microbial contamination of impression materials is very scarce. The sterility of dental 
materials as delivered to the dentist from manufacturers has very little information about infection control during processing and packaging. Several studies have addressed preventing the transmission of microorganisms via impression materials after exposure to the patient's oral fluids. ${ }^{3-6,16-18}$ One aspect that has been neglected is the risk from contamination of dental materials during manufacturing and packaging process. Contamination standards on expendable dental materials are not available. Until today, very few measures have been taken by manufacturers to avoid such contamination.

Gates et al found bacterial contamination in all the tested sealed bottles of four commercially available denture adhesives. $^{7}$ Moghadam et al found aerobic bacterial contamination in approximately $30 \%$ of the samples in four dental materials. ${ }^{8}$ After that, other studies were performed on irreversible hydrocolloid pointing out the contamination of various brands, including those containing antimicrobial agents. ${ }^{9-11 .}$

The purpose of this study was to evaluate factory-sealed containers of three different commercially available irreversible hydrocolloid impression materials for their microbial contamination and the type of microorganisms present.

\section{MATERIALS AND METHODS}

The study was conducted using testing equipment in the Department of Microbiology, JNMC, Belgaum and Department of Prosthodontics and Crown and Bridge, KLE's Institute of Dental Sciences, Belgaum. Three sealed packets, each of three different brands of most commonly used commercially available irreversible hydrocolloid impression materials, were purchased from the manufacturers. The tested materials were hydrogum (Batch No. A0243B, Heraues Kulzer, Germany), plastalgin (Batch No. 08801623001, Septodont, Italy), algitex (Batch

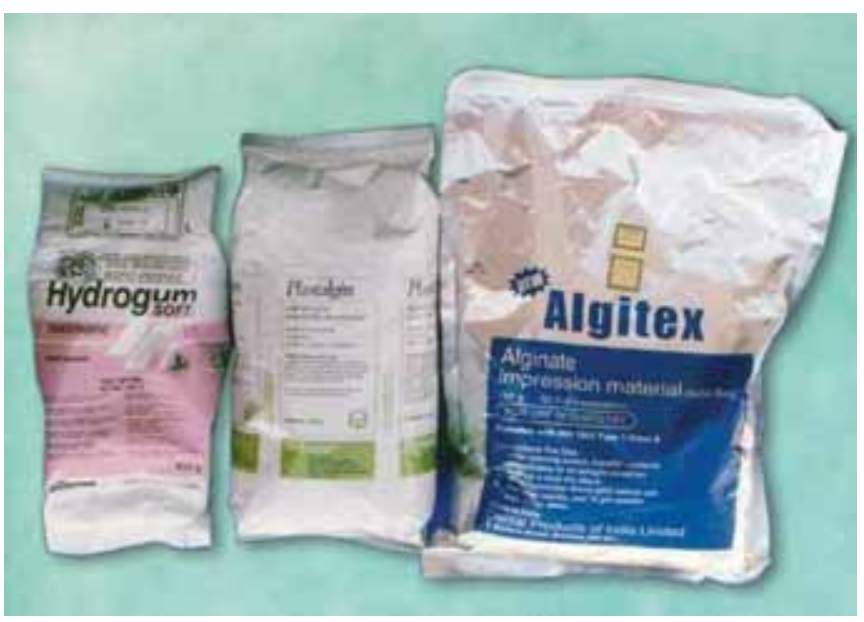

Fig. 1: Materials used
No.Y3213, DPI, India) (Fig. 1). The packaging of all the packets was examined to ensure that they were free from any violation.

With full aseptic precautions, the sealed packets were opened in a laminar flow hood and four samples measuring 0.06 gm each were removed from the top, middle and bottom layers with the help of sterile instruments. Individual chocolate agar plates (for culture of aerobic microorganisms) were then inoculated by streaking procedure with six samples from each packet; two from the top, two from the middle and two from the bottom. The remaining samples were placed in tubes of thioglycolate broth (for culture of anaerobic microorganisms) and were vortexed for 10 seconds to disperse the irreversible hydrocolloid. For each sample, appropriate parallel controls of uninoculated chocolate agar plates and the same number of uninoculated thioglycolate broths served as negative controls.

Ten chocolate agar plates and 10 thioglycolate broth tubes were challenged with a mixture of four isolates (E. coli ATCC No. 25922, Staphylococcus aureus ATCC No. 29213, Pseudomonas aeruginosa ATCC No. 27853, and Streptococcus faecalis ATCC No. 29212) to ensure that the media supported growth of microorganisms. ${ }^{12}$

All chocolate agar plates were labeled individually according to the tested material and placed in an incubator at $37^{\circ} \mathrm{C}$ for 7 days. After 7 days, they were observed for presence or absence of microbial growth (Fig. 2). The thioglycolate broths inoculated with samples were incubated at $37^{\circ} \mathrm{C}$ for 5 days in an incubator. After 5 days, any sample showing turbidity was again subcultured to chocolate agar and blood agar plates which were kept in McIntosh Fildes jar and incubated for two days at $37^{\circ} \mathrm{C}$ in an incubator (Fig. 3).

After incubation, the colony forming units (CFU) were counted based on the values obtained for $0.06 \mathrm{gm}$ and the organisms were identified based on colonial morphology, Gram's stain, coagulase test, hemolytic reactions and pigmentations.

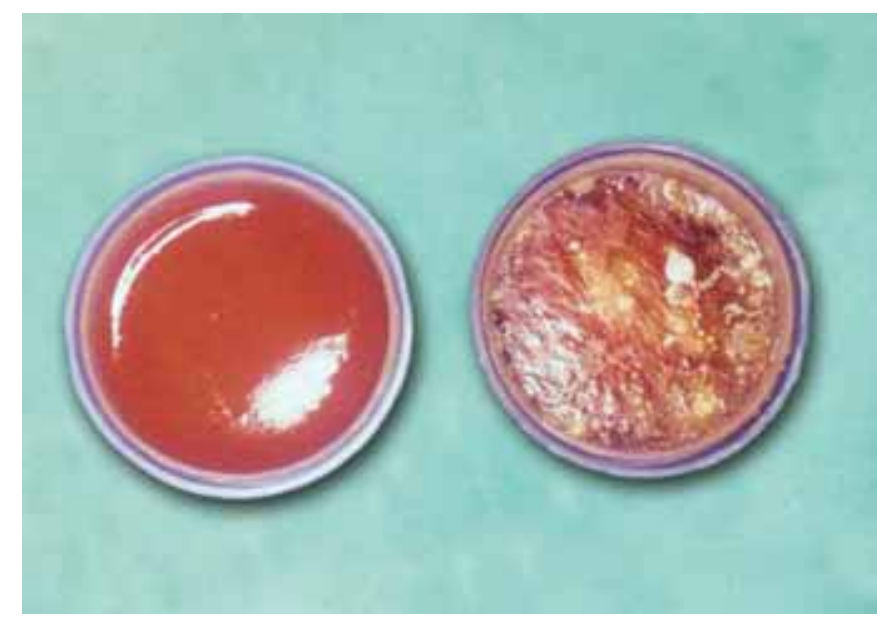

Fig. 2: Chocolate agar plates (before and after culture) 


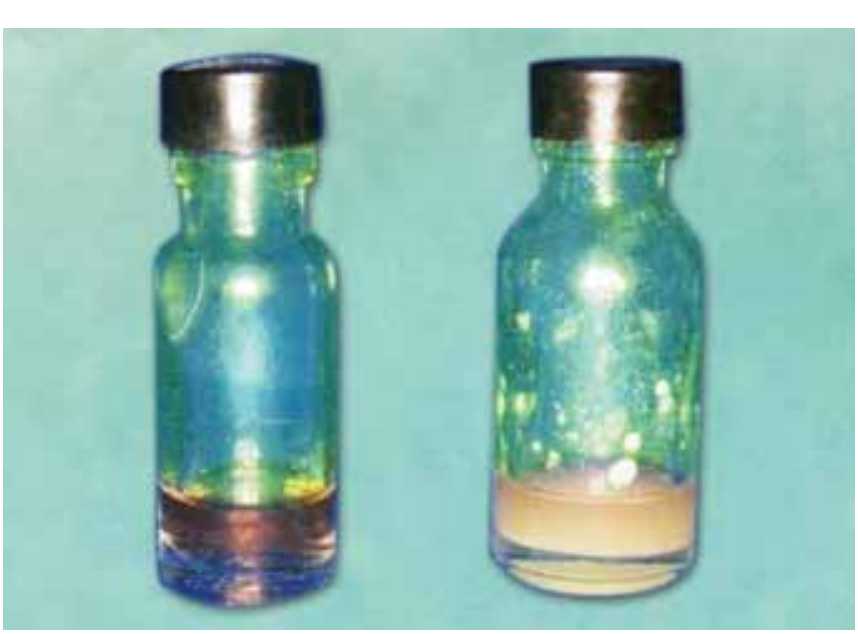

Fig. 3: Thioglycolate broth (before and after culture)

The number of positive results in each category of media, the location of the sample, and the brand was divided by the total number of samples to arrive at the contamination frequency. This factor was compared with the data from the control groups and other experimental groups using Student's t-test for statistical significance to determine the results.

\section{RESULTS}

All culture media inoculated as positive controls with microorganisms exhibited appropriate growth. All uninoculated culture media were void of growth. None of the negative controls exhibited any growth.

There was an average of 44 CFUs per gram of contaminated material. Plastalgin showed the least at 25 CFUs per gram, followed by hydrogum which had 32 CFUs per gram and algitex at 74 CFUs per gram. The number of CFUs per gram of the sample in chocolate agar plates and thioglycolate broth tubes is given in Graphs 1 and 2.

The spectrum of various microorganisms in chocolate agar and thioglycolate broths is shown in Graphs 3 and 4. Inoculated chocolate agar plates showed an average contamination frequency of $96.2 \%$ while that of thioglycolate broth tubes resulted in a contamination average of $82.8 \%$, the difference was not statistically significant. There was no significant difference in contamination frequency between the top, middle and bottom layers of samples from the packets (89.8, 88.6 and 93\% respectively) as shown in Table 1 . The overall contamination average (top, middle and bottom; both chocolate agar and thioglycolate broth) was plastalgin-88\%, algitex-94.4\%, hydrogum-88\% (Table 2).

\section{DISCUSSION}

Dental practitioners and patients are subject to notable risks with respect to infectious diseases, which can be spread by

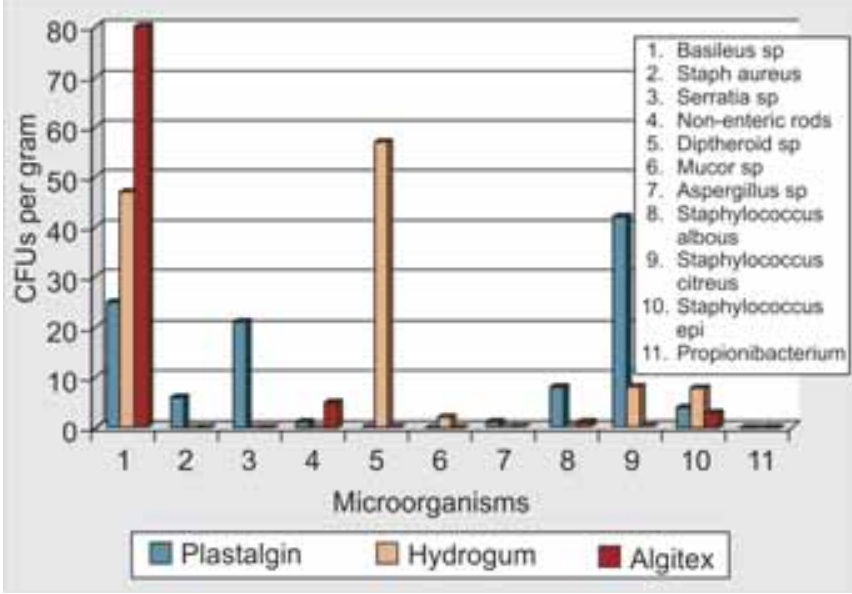

Graph 1: Chocolate agar contamination concentration for each brand (number of CFUs per gram)

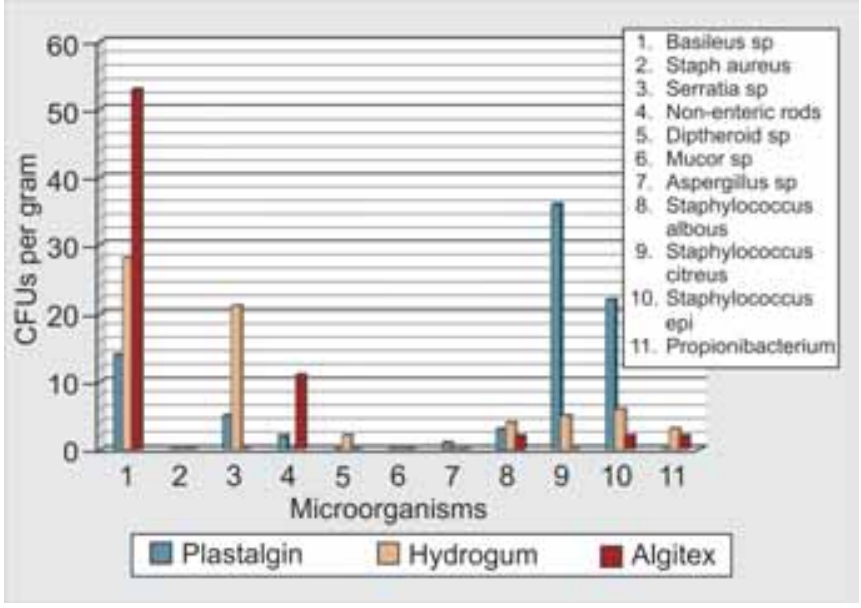

Graph 2: Thioglycolate broth contamination concentration for each brand (number of CFUs per gram)

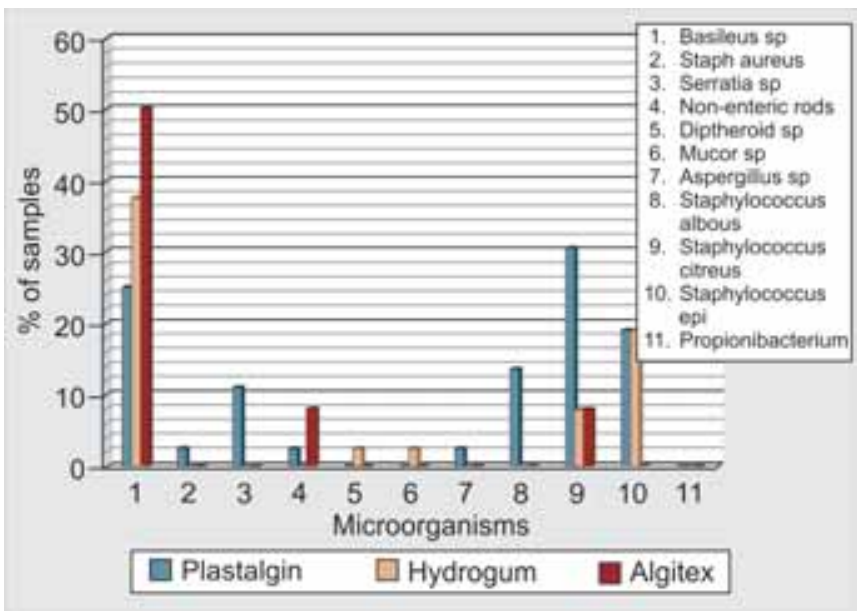

Graph 3: Chocolate agar contamination frequency (\%)

saliva or blood from contaminated impression material, particularly irreversible hydrocolloid impression material. Irreversible hydrocolloid impression material is forcefully positioned against oral mucosa while making the impression. 


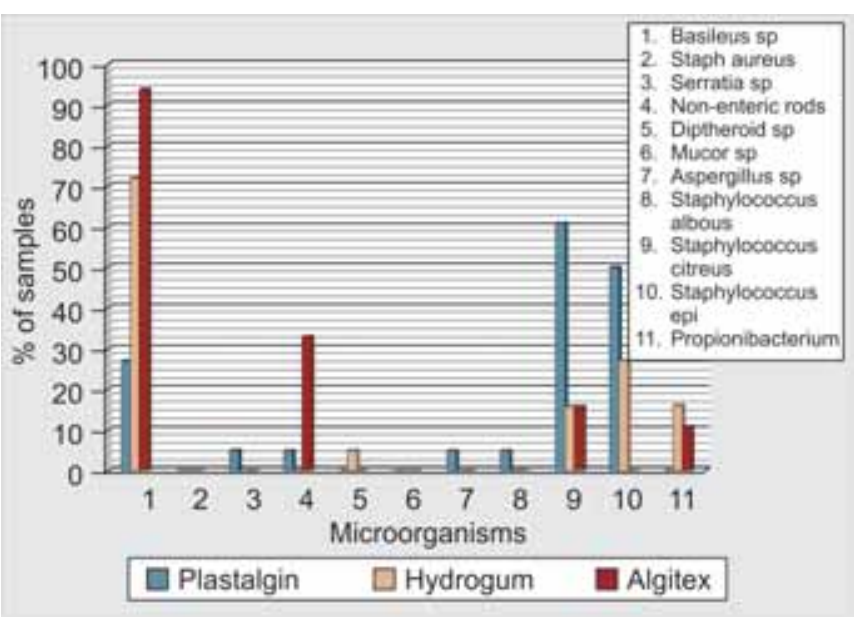

Graph 4: Thioglycolate broth contamination frequency (\%)

\begin{tabular}{|c|c|c|c|c|}
\hline \multicolumn{5}{|c|}{$\begin{array}{l}\text { Table 1: Comparison of contamination frequencies }(\%) \text { at different } \\
\text { layers of the three brands (both chocolate agar and thioglycolate } \\
\text { broth media) }\end{array}$} \\
\hline \multirow[t]{2}{*}{ Layer } & \multicolumn{3}{|c|}{ Brand } & \multirow[t]{2}{*}{ Total } \\
\hline & Plastalgin & Algitex & Hydrogum & \\
\hline Top & $87.5 \%$ & $92.5 \%$ & $89.7 \%$ & $89.8 \%$ \\
\hline Middle & $88.2 \%$ & $90.4 \%$ & $88.1 \%$ & $88.6 \%$ \\
\hline Bottom & $92 \%$ & $95.4 \%$ & $93.4 \%$ & $93.2 \%$ \\
\hline
\end{tabular}

\begin{tabular}{|c|c|c|c|}
\hline & \multicolumn{3}{|c|}{ Brand } \\
\hline & Plastalgin & Algitex & Hydrogum \\
\hline$\%$ of contaminated samples & $88 \%$ & $94.4 \%$ & $88 \%$ \\
\hline
\end{tabular}

A visual study of the impression immediately after removal from the mouth often reveals blood clinging to the material. ${ }^{13}$ Blood is a well-recognized vector of pathogenic microorganisms and there are few if any dental procedures in which blood contamination is not a risk. ${ }^{14}$ There is a chance of potentially inoculating microorganisms if the material is contaminated. Because alginates have polysaccharide structures similar to those of agar which is an excellent substrate for microorganisms, it seems unlikely that alginate powders would be free from microorganisms. Hence, the use of contaminated impression materials may represent an additional risk of inoculation of microorganisms and, consequently, of occurrence of diseases in immunocompromised patients. Though various studies have shown that irreversible hydrocolloids may contain viable microorganisms; however, very few measures have been taken by manufacturers to address this issue. ${ }^{9-11,15,19}$ No contamination standards are known to exist for expendable dental materials. There is very little information regarding the sterility of dental materials as delivered to the dentist from the manufacturer. Sterilization methods should be incorporated, such as irradiation with gamma rays at the end of production. Nonetheless, such method requires a previous knowledge concerning microbial load, both qualitatively and quantitatively, in order to determine the doses to be applied. To improve the basis for risk assessment, and to find a suitable strategy for cross- contamination risks, there is a need to study to determine microbial contamination in impression materials, particularly alginate.

Murray found all study samples contaminated with microorganisms which could pose danger to immunocompromised patients. ${ }^{16}$ Moghadam et al studied incidence of aerobic bacterial contamination in 12 different dental materials and found viable organisms in samples of 4 of 12 different materials. ${ }^{8}$ Rice compared four brands of irreversible hydrocolloid impression materials and concluded that viable organisms were present in 50\% to $100 \%$ of the samples. ${ }^{9}$ In another study by Rice on bacterial contamination of unopened packets of irreversible hydrocolloid impression material and gingival retraction cord, a statistically significant number of alginate samples were found to be microbiologically contaminated. ${ }^{10}$ Rice et al also studied antimicrobial alginates for contamination and found that $12.5 \%$ of the samples contained viable organisms. ${ }^{11}$

A statistically significant (90\%) percentage of samples from the three brands of irreversible hydrocolloid impression material tested showed microbial contamination irrespective of the brand or batch. This is in agreement with findings of Rice et al who found viable organisms in $100 \%$ of the samples. ${ }^{9,11}$ The chocolate agar media showed an average contamination frequency of $96.2 \%$ of samples, while that of thioglycolate broths showed a contamination average of $82.8 \%$. This may have been due to the clumping of irreversible hydrocolloid in the thioglycolate broths.

With respect to the microorganisms isolated, the spectrum of contaminants in chocolate agar found Bacillus sp (72\%) which are common environmental contaminants of low virulence. These were followed by Staphylococcus citreus (31\%), Staphylococcus epidermidis (26\%), Staphyloccus albus (9\%), Serratia sp (7.4\%) (Fig. 4), Mucor $(1.8 \%)$ and Diphtheroids $(1.8 \%)$ which are opportunistic pathogens. These findings are similar to those detected in studies carried out by Rice and Gates. ${ }^{9,11,7}$ Staphylococcus aureus (1.8\%) and Aspergillus (1.8\%) which are moderately virulent organisms were also isolated and have been documented as a cause of infection in immunocompromised patients. ${ }^{13}$ Similar findings were seen in studies by Rice, Powell and Montgomery who found these organisms in less than $1 \%$ of the samples. ${ }^{9,17,18}$ 


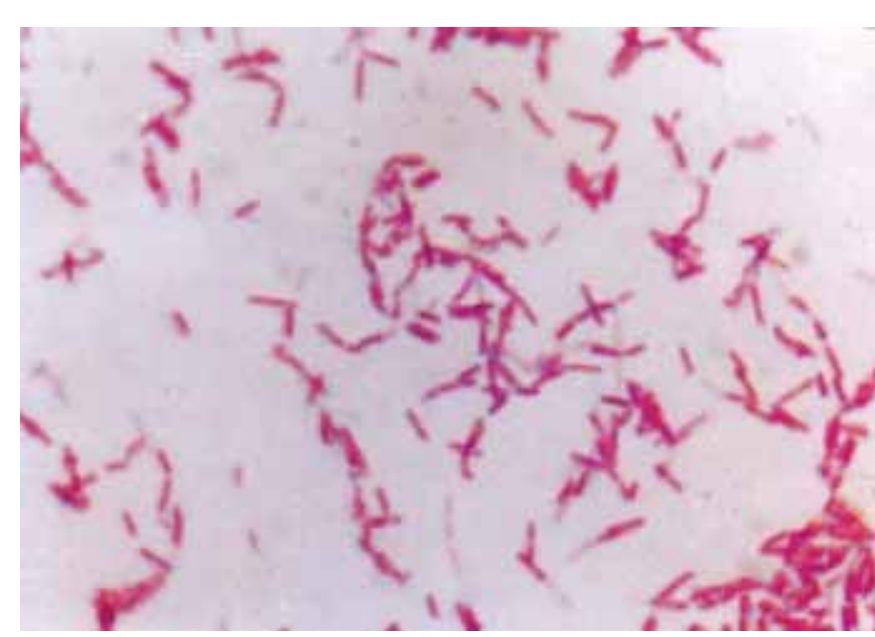

Fig. 4: Serratia species (gram-negative bacilli)

Organisms detected in the thioglycolate broths were Bacillus sp (65\%), Staphylococcus citreus (31\%), Staphylococcus epidermidis (26\%), Staphylococcus albus (1.8\%), Nonenteric gram-positive rods (13\%)and Aspergillus (1.8\%) all discussed previously. Propionibacterium (9\%) which is an anaerobic skin commensal of low virulence was also found.

Colony forming units per gram of impression material was also measured and an average of $44 \mathrm{CFUs/gm} \mathrm{of}$ irreversible hydrocolloid impression material was found, while a study by Rice et al found 12 to $82 \mathrm{CFUs} / \mathrm{gm} .{ }^{9}$ Algitex showed the highest CFUs/gm at 74, followed by hydrogum at $32 \mathrm{CFUs/gm}$ and plastalgin at $25 \mathrm{CFUs/gm}$. The difference found was not statistically significant. Overall, the percentage of contamination for each brand was $94.4 \%$ for algitex, $88 \%$ for hydrogum and $88 \%$ for plastalgin.

Regarding the layer-wise comparison of contamination frequency, no significant difference was found in the frequency of contamination in the top, middle and bottom layers of the irreversible hydrocolloid packets respectively. This finding is again in accordance with studies by Rice et al. ${ }^{9,11}$

\section{CLINICAL SIGNIFICANCE}

Clinically, the average clinical material required for an alginate impression is $18 \mathrm{gms}$ which is 300 times the testing sample. This would yield an average concentration of 1200 organisms per average clinical sample of material for all the materials combined which is higher than in previous studies. ${ }^{9,11}$

Most of the organisms found were avirulent members of the environmental flora. McGhee et al cautioned that the compromised patient may have an altered flora and be susceptible to infection with less usual organisms and ordinary dental procedures. In severely immunocompromised hosts, organisms that are considered avirulent could nonetheless be pathogenic. A small percentage of samples were found to contain virulent organisms that may represent a possible risk to all patients. Routine use of contaminated dental materials in immunocompromised patients could lead to inoculation.

With regards to the origin of the contamination present, it may be due to the materials used, to the manufacturing process, transportation, and even caused iatrogenicaly.This may be due to the fact that, even if the alginates were free of contamination at first, there would certainly still be a possibility of contamination because the material is kept in a large package, whose content is sufficient to perform many impressions. By opening the container, microorganisms from the environment could be introduced into it, which would make the material inadequate for further use from a microbiological point of view.

\section{CONCLUSION}

All the irreversible hydrocolloids tested showed microbiological contaminants with brands varying appreciably in spectrum and concentration of contaminants. Most of the organisms were low virulent contaminants that may represent a hazard to immunocompromised patients. A small percentage of samples were found to contain virulent organisms that may represent a possible risk to all patients. More attention should be paid to the microbiological quality and sterility of commercially available alginates and to determine, by performing other studies, if these materials should be sterile or should be allowed a microbiological limit, both qualitatively and quantitatively. It is also recommended that the packages be presented with a singleuse size (for a single impression) in order to preserve the microbiological quality of the material. ${ }^{19}$

\section{REFERENCES}

1. McGhee JR, Michalek SM, Cassel GH. Dental Microbiology. New York; Harper Row 1982.

2. Gurevich I Infection control: Applying theory to clinical practice. In: Block SS. Disinfection, sterilization and preservation (4th edn) Malvern; Lea and Febiger 1991;655-62.

3. Cserna A, Crist RL, Adams AB, Dunning DG. Irreversible hydrocolloids: A comparison of antimicrobial efficacy. J Prosthet Dent 1994;71:387-89.

4. Dowell TB. The problem of cross infection in dentistry. BDAdental health and services committee workshop. Br Dent Journal 1986;160:131-34.

5. Setcos JC, Gerstenblatt R, Palenik CJ, Hinoura K. Disinfection of a polyether dental impression material. Journal of Dental Research 1985; 64:244.

6. Johnson GH, Drennon DG, Powell GL. Accuracy of elastomeric impression materials disinfected by immersion. JADA 1988;116: 525-30. 
7. Gates WD, Goldschmitt M, Kramer D. Microbial contamination in four commercially available denture adhesives. J Prosthet Dent 1994;71:154-58.

8. Moghadam B, Gier RE, Cobb CM. Aerobic bacterial contamination in dental materials. Oral Surg Oral Med Oral Pathol 1990;70(4):537-39.

9. Rice CD, Dykstra MA, Gier RE, Cobb CM. Microbial contamination in four brands of irreversible hydrocolloid impression materials. J Prosthet Dent 1991;65(3):419-23.

10. Rice CD, Dykstra MA, Gier RE. Bacterial contamination in irreversible hydrocolloid impression material and gingival retraction cord. J Prosthet Dent 1991;65(4):496-99.

11. Rice CD, Dykstra MA, Feil PH. Microbial contamination in two antimicrobial and four control brands of alginate impression material. J Prosthet Dent 1992;67(4):535-40.

12. Topley, Wilson. Principles of bacteriology, virology and immunity (6th ed). London; Edward Arnold.

13. Driezen S, McCredie KB, Keating MJ, Bodey GP. Oral infections associated with chemotherapy in adults with acute leukemia. Postgrad Med 1982;71:133-46.

14. Martin MV. Infection control in the dental environment. London; Martin Dunitz 1991.

15. Palenik CJ, Setcos JC, Miller CH. Antimicrobial activity of a disinfectant containing alginate impression material. Journal of Dental Research 1990;69:348-53.

16. Murray JP, Slack GL. Sources of bacterial contamination in every day dental practice. Br Dent Journal 1957;15: 184-86.

17. Powell GL, Fenn JP. Cavity varnish and bacterial cross contamination. Oper Dent 1986;11:90-94.
18. Montgomery S. Chemical decontamination of gutta percha cones with polyvinyl pyrrolidone-iodine. Oral Surgery 1971;31: 258-66.

19. Warwick EF. Preventing microbial contamination in manufacturing. Cosmetics and Toiletries 1993;108(10): 77-82.

\section{ABOUT THE AUTHORS}

\section{Rakshith Guru}

Professor, Department of Prosthodontics, Hazaribagh College of Dental Sciecnes, Hazaribagh, Jharkhand, India

\section{Mohammed Saleem}

Professor, Department of Prosthodontics, KGF Dental College, Kolar Karnataka, India

\section{Rajeev Singh}

Professor, Department of Prosthodontics, DY Patil Institute of Dental Sciences, Mumbai, Maharashtra, India

\section{Anand Patil}

Reader, Department of Prosthodontics, Mansarovar College of Dental Sciences, Bhopal, Madhya Pradesh, India

\section{CORRESPONDING AUTHOR}

Rakshith Guru, 11, 1st Cross, Next to CMR School, Chennakeshava Layout, KK Halli, Bengaluru-560084, Karnataka, India, Phone: 9900197517 e-mail-rakshith77@yahoo.com 